\title{
An energy-based method for analyzing instrumented spherical indentation experiments
}

\author{
Wangyang Ni \\ Materials and Processes Laboratory, General Motors Research and Development Center, \\ Warren, Michigan 48090, and Department of Chemical Engineering and Materials Science, \\ Michigan State University, East Lansing, Michigan 48824 \\ Yang-Tse Cheng ${ }^{\text {a) }}$ \\ Materials and Processes Laboratory, General Motors Research and Development Center, \\ Warren, Michigan 48090 \\ Che-Min Cheng \\ Institute of Mechanics, Chinese Academy of Sciences, Beijing 100080, People's Republic of China \\ David S. Grummon \\ Department of Chemical Engineering and Materials Science, Michigan State University, \\ East Lansing, Michigan 48824
}

(Received 16 June 2003; accepted 23 September 2003)

Using dimensional analysis and finite element calculation, we studied spherical indentation in elastic-plastic solids with work hardening. We report two previously unknown relationships between hardness, reduced modulus, indentation depth, indenter radius, and work of indentation. These relationships, together with the relationship between initial unloading stiffness and reduced modulus, provide an energy-based method for determining contact area, reduced modulus, and hardness of materials from instrumented spherical indentation measurements. This method also provides a means for calibrating the effective radius of imperfectly shaped spherical indenters. Finally, the method is applied to the analysis of instrumented spherical indentation experiments on copper, aluminum, tungsten, and fused silica.

\section{INTRODUCTION}

Since the inception by Brinell about 100 years ago, spherical indentation techniques have become industry standard methods for measuring the hardness of materials. ${ }^{1-4}$ Recent years have seen increasing interest in instrumented spherical indentation techniques for measuring the mechanical properties of materials across multiple length scales. By analyzing the load-displacement curves, several authors have suggested methods of obtaining hardness, elastic modulus, stress-strain relationships, and residual stresses from instrumented spherical indentation measurements. ${ }^{5-12}$ However, most of the previously proposed methods depend on the estimation of the contact area under the spherical indenter, which is difficult especially when the surface around the indenter exhibits "piling-up." "13-16

a) This author was an editor of this focus issue during the review and decision stage. For the $J M R$ policy on review and publication of manuscripts authored by editors, please refer to http:// www.mrs.org/publications/jmr/policy.html
By extending a recently developed scaling approach $^{17,18}$ to spherical indentation in elastic-plastic solids with work-hardening, we reveal, in this paper, two previously unknown relationships between hardness, reduced modulus, indentation depth, indenter radius, and work of indentation. Together with a well-known relationship ${ }^{5}$ between reduced modulus, initial unloading slope, and contact area, we propose an energy-based method of determining the contact area, reduced modulus, and hardness of materials from instrumented spherical indentation experiments. This method also provides a way of calibrating the effective radius of imperfect spherical indenters. The validity of the method is tested by instrumented spherical indentation experiments on copper, aluminum, tungsten, and fused silica. These newly found relationships also provide fresh insights into contact problems that are ubiquitous in many science and engineering areas, including friction, wear, as well as macro- and nanoscale mechanical forming processes.

\section{DIMENSIONAL AND FINITE ELEMENT ANALYSIS}

We consider a three-dimensional, rigid, spherical indenter of radius, $R$, indenting into an isotropic elastic- 
plastic material with work hardening [Fig. 1(a)]. The stress-strain $(\sigma-\epsilon)$ curves of the solid under uniaxial tension are given by

$$
\begin{aligned}
& \sigma=E \epsilon, \text { for } \epsilon \leqslant Y / E, \\
& \sigma=K \epsilon^{n}, \text { for } \epsilon>Y / E,
\end{aligned}
$$

where $E$ is the Young's modulus, $Y$ is the initial yield stress, $K$ is the strength coefficient, and $n$ is the strainhardening exponent. To ensure continuity at the initial yield stress, we note $K=Y(E / Y)^{n}$. Consequently, $E, Y$, and $n$ are sufficient to describe the uniaxial stress-strain relationship. The friction coefficient at the contact surface between the indenter and the solid is assumed to be zero.

During loading, the two dependent variables, force $\left(F_{l}\right)$ and contact depth $\left(h_{c}\right)$, must be functions of all six independent governing parameters, $E$, Poisson's ratio (v), $Y$,
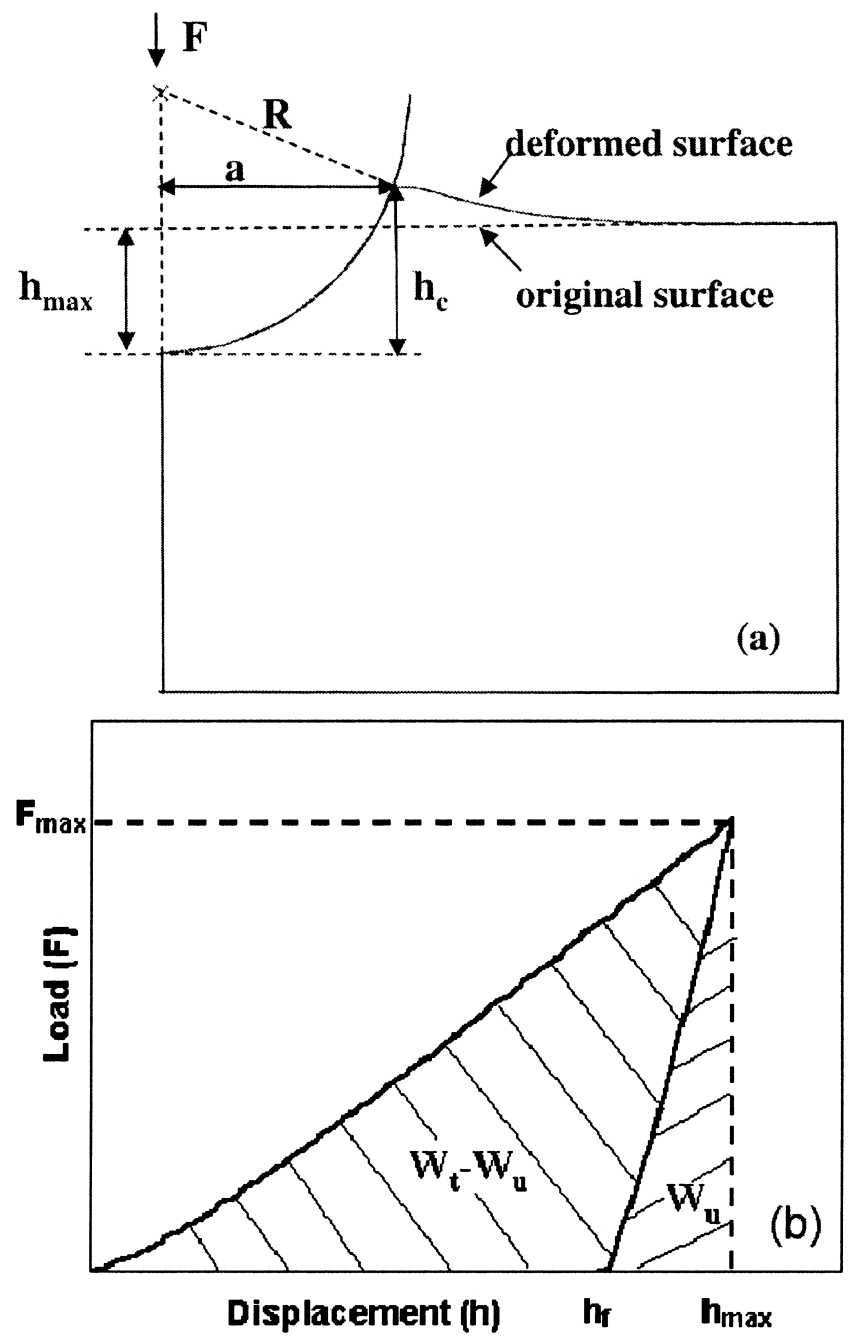

FIG. 1. (a) Schematic illustration of spherical indentation, and (b) loading-unloading curves and definition of the irreversible work, $W_{t}-W_{u}$, and reversible work, $W_{u}$. $n$, indenter displacement $(h)$, and $R$. Applying the $\Pi$ theorem in dimensional analysis, ${ }^{19}$ we obtain

$$
\begin{aligned}
& F_{l}=E R^{2} \prod_{\alpha}\left(\frac{Y}{E}, v, n, \frac{h}{R}\right), \\
& h_{c}=R \prod_{\beta}\left(\frac{Y}{E}, v, n, \frac{h}{R}\right),
\end{aligned}
$$

where $\Pi_{\alpha}=F_{l} / E R^{2}$ and $\Pi_{\beta}=h_{c} / R$ are two dimensionless functions of four dimensionless parameters: $Y / E, v$, $n$, and $h / R$.

During unloading, the force on the indenter $\left(F_{u}\right)$ is a function of all seven independent parameters, $E, v, Y, n$, $h, R$, and indenter displacement at maximum load $\left(h_{\max }\right)$ because unloading occurs after the indenter reaches $h_{\max }$. Again, applying the $\Pi$ theorem in dimensional analysis, ${ }^{19}$ we obtain

$$
F_{u}=E R^{2} \prod_{\gamma}\left(\frac{Y}{E}, v, n, \frac{h}{R}, \frac{h_{\max }}{R}\right),
$$

Equations (2) and (4) can formally be integrated with respect to displacement to obtain expressions for the total work, $W_{t}$, and reversible work, $W_{u}$, defined as the areas under the respective loading and unloading curves [Fig. 1(b)]. Furthermore, we have shown ${ }^{20}$ (see the Appendix) that $h_{c} / h_{\max }, h_{f} / h_{\max },\left(W_{t}-W_{u}\right) / W_{t}$, and ratio of hardness to reduced modulus, $H / E^{*}$ where $E^{*}=E /(1-$ $v^{2}$ ), are all dimensionless functions of $Y / E, v, n$, and $h_{\text {max }} / R$. It is therefore interesting to investigate the relationships between $\left(W_{t}-W_{u}\right) / W_{t} h_{f} / h_{\max }$, and $H / E^{*}$, as well as the degree of "piling-up" (i.e., $h_{c} / h_{\max }>1$ ) and "sinking-in" $\left(h_{c} / h_{\max }<1\right)$ as a function of materials properties $(Y / E, \nu$, and $n)$ and indentation depth $\left(h_{\max } / R\right)$.

Because there is no analytical solution for spherical indentation in elastic-plastic materials, a finite element analysis was used to study the problem. In this work, an extensive finite element computation was carried out using ABAQUS (version 6.2, Hibbitt, Karlsson \& Sorensen, Inc., Pawtucket, RI) to calculate $h_{c} / h_{\max }, h_{f} / h_{\max }$, $\left(W_{t}-W_{u}\right) / W_{t}$, and $H / E^{*}$. The radius of the rigid indenter was $1 \mu \mathrm{m}$. The Young's modulus was $200 \mathrm{GPa}$, and Poisson's ratio was 0.2 . The strain-hardening exponent, $n$, was chosen to be $0.1,0.3$, and 0.5 , respectively. For each strain-hardening exponent, five different $Y / E$ values were used, namely, 0.002, 0.005, 0.01, 0.025, and 0.1, which represent materials from soft metals to hard ceramics. For each combination of $n$ and $Y / E$, computations were carried out for several values of $h_{\max }$, allowing the evaluation of the scaling functions for a range of $h_{\max } / R$ values from 0.05 to 0.5 .

The rate-independent, incremental theory of plasticity in ABAQUS was used in the finite element calculations. In particular, the plasticity theory uses the von Mises' yield surface model with associated plastic flow rule. 
Because of the axisymmetry of the problem, twodimensional mesh and boundary conditions, similar to those in Refs. 17 and 18, were adapted. The smallest element, which lies in the contact region, is $25 \mathrm{~nm}$. The load-displacement curves as well as contact area were obtained directly from the finite element calculations. The residual indentation depth, $h_{f}$, and the maximum load, $F_{\text {max }}$, were obtained from the load-displacement curves for each given $h_{\max }$. The total work, $W_{t}$, and reversible work, $W_{u}$, are obtained by integrating the loading and unloading curves, respectively.

\section{A. Piling-up/sinking-in in spherical indentation}

The ratio of $h_{c} / h_{\max }$ is used to evaluate the degree of the piling-up and sinking-in. The calculated values of $h_{c} / h_{\max }$ for various materials at different indentation depths are shown in Fig. 2. It is evident that, in general, $h_{c} / h_{\max }$ depends on $h_{\max } / R, n$, and $Y / E$ for spherical indentation in elastic-plastic solids with work hardening. For materials with small values of $Y / E$ and $n$ (i.e., typical work-hardened metals), sinking-in occurs at small indentation depth whereas piling-up occurs at large indentation depth [Figs. 2(a) and 2(b)]. For materials with large values of $Y / E$ and $n$, sinking-in is observed for all indentation depths [Fig. 2(c)]. These observations can be understood by the fact that sinking-in always occurs for Hertzian elastic contacts, whereas piling-up occurs for rigid-plastic contacts. ${ }^{2}$ Thus, the ratio of "elastic component" to "plastic component" of deformation decreases with increasing $h_{\max } / R$ and decreasing values of $Y / E$ and $n$ for spherical indentation in elastic-plastic solids with work hardening.

It is instructive to note that the degree of piling-up and sinking-in is independent of depth for conical and pyramidal indentation in the same class of solids. This depth independence is the consequence of the absence of a length parameter associated with ideally sharp conical and pyramidal indenters. ${ }^{17}$ It is also instructive to compare the current numerical results with some early experimental work by Norbury and Samuel, ${ }^{13}$ who believed that piling-up and sinking-in depended on the work-hardening exponent, $n$, only. Their observation was primarily based on indentation in metals (i.e., small values of $Y / E$ with $n$ values from 0.0 to 0.5 ) where the effect of $n$ is dominant. In general, however, the degree of piling-up and sinking-in depends on $Y / E, n$, and $h_{\max } / R$ which makes the determination of contact area under load difficult using conventional methods. In the following analysis, we show that it is possible to circumvent this difficulty of estimating contact area using an energy-based method derived from a scaling relationship between $H / E^{*}$ and $\left(W_{t}-W_{u}\right) / W_{t}$.
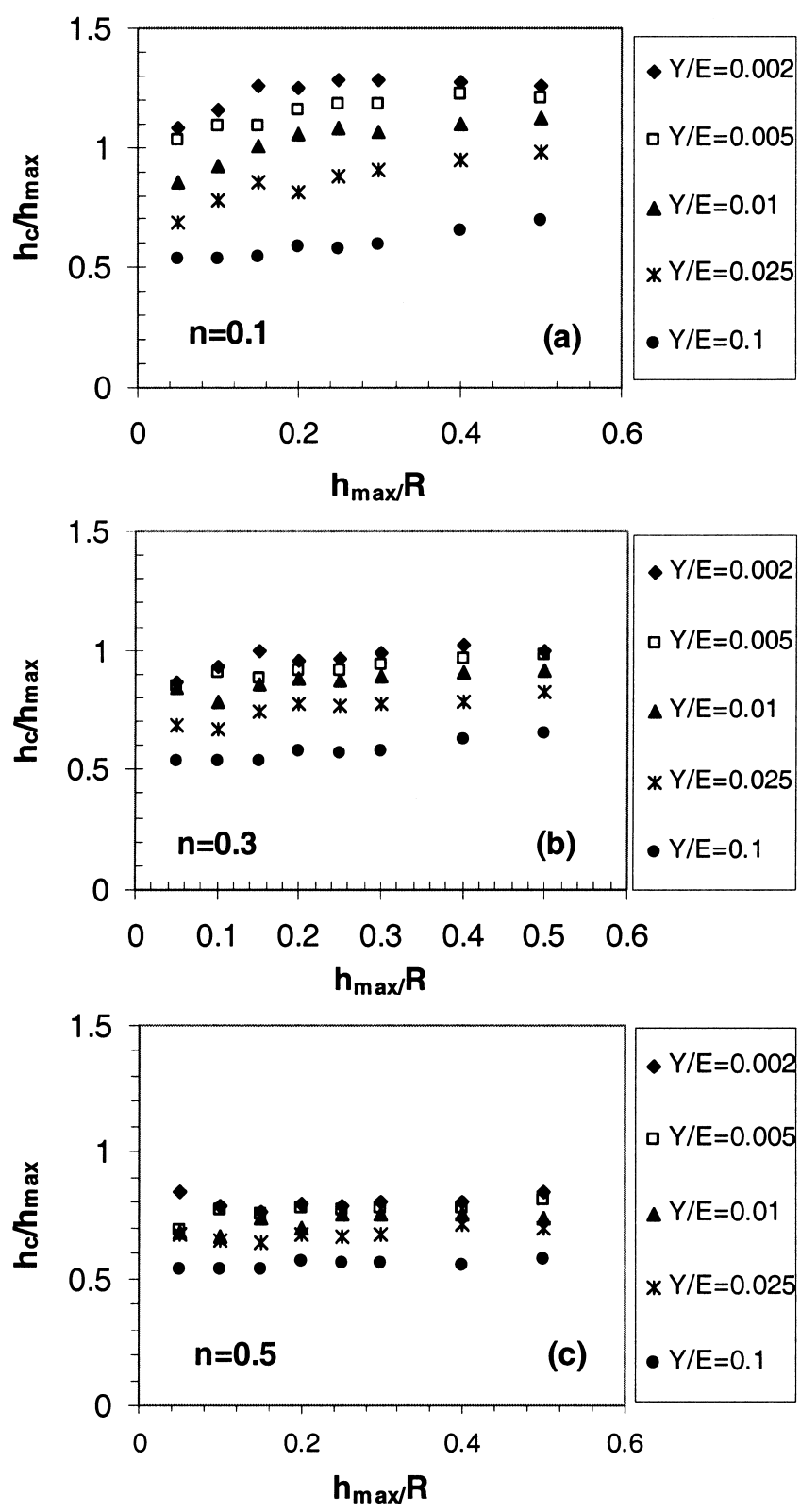

FIG. 2. Degree of piling-up and sinking-in as a function of $h_{\max } / R, n$, and $Y / E$ in spherical indentation modeling. (a) $n=0.1$, (b) $n=0.3$, and (c) $n=0.5$.

\section{B. Relationship between $h_{f} / h_{\max }$ and $\left(W_{t}-W_{u}\right) / W_{t}$}

The representative load-displacement curves, obtained by finite element calculations, of a material with $Y / E=$ $0.025, v=0.2$, and $n=0.5$ at various depths are shown in Fig. 3. By analyzing the load-displacement curves, we observed a relationship between $\left(W_{t}-W_{u}\right) / W_{t}$ and $h_{f} /$ $h_{\max }$, which is shown in Fig. 4. This relationship can, from a least squares fit, be written as

$$
\frac{h_{f}}{h_{\max }}=\frac{W_{t}-W_{u}}{W_{t}}
$$

Equation (5) shows that the degree of permanent deformation, measured by the ratio of residual depth to 


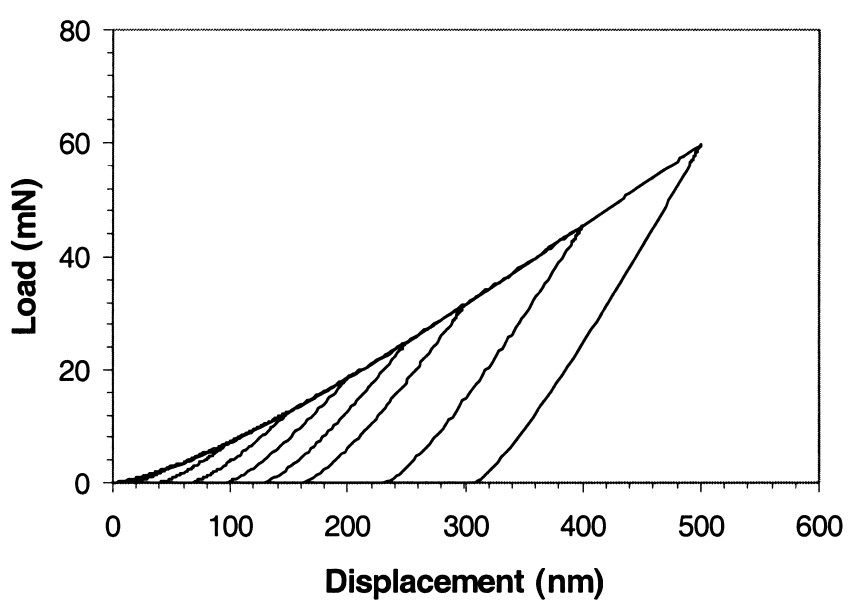

FIG. 3. Calculated load-displacement curves of a material with $Y / E$ $=0.025, v=0.2$, and $n=0.5$ at various indentation depths.

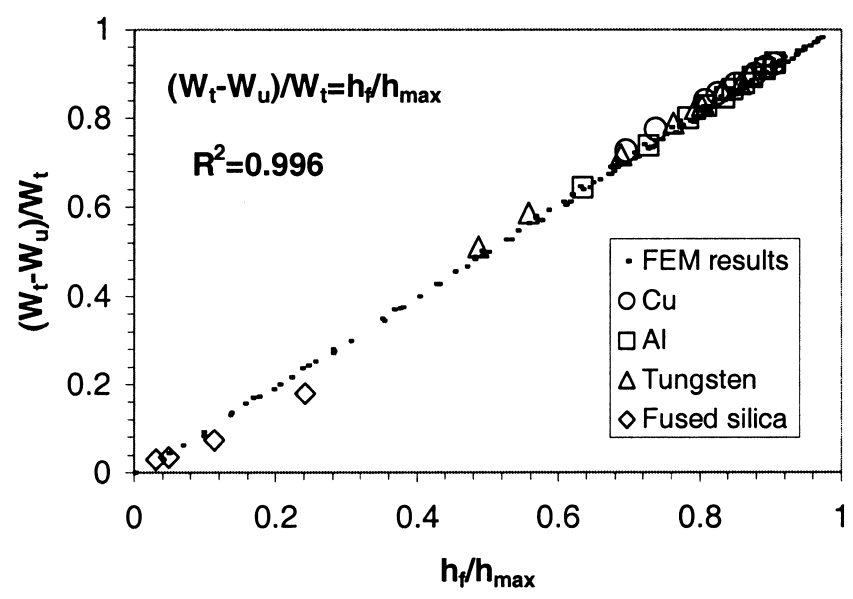

FIG. 4. A linear relationship exists between $h_{f} / h_{\max }$ and $\left(W_{t}-W_{u}\right) / W_{t}$ : finite element results and experimental data.

maximum indenter displacement, $h_{f} / h_{\max }$, is simply related to the ratio of irreversible work to total work, $\left(W_{t}-\right.$ $\left.W_{u}\right) / W_{t}$. The measurement of one leads to the measurement of the other. In practice, however, the determination of the work can be made more accurately than the measurement of $h_{f}$, as the former is from the integration of loading-displacement curves, whereas the latter is from the estimation of a single point on the unloading curve. Furthermore, this relationship is "universal" because it does not depend on the details of the mechanical behavior of solids, such as $E, Y$, and $n$. Nor does the relationship depend on the indenter radius, $R$, or indentation depth, $h$. This new relationship is analogous to a relationship previously established for conical and pyramidal indentation in elastic-plastic solids with work hardening. ${ }^{18,21}$ Together, they demonstrate that a simple linkage exists between the work of indentation and deformation which is independent of the details of material properties and indenter geometry.

\section{Relationship between $H / E^{*}$ and $\left(W_{t}-W_{u}\right) / W_{t}$}

An approximately linear relationship between $H / E^{*}$ and $\left(W_{t}-W_{u}\right) / W_{t}$ for spherical indentation in elasticplastic solids is revealed in Fig. 5. For each fixed $h_{\max } / R$, this relationship can be expressed as

$$
\frac{\left(W_{t}-W_{u}\right)}{W_{t}}=B \frac{H}{E^{*}}+1
$$

where $B$ is obtained from a least squares fit to the linear relationships in Fig. 5. Furthermore, the value of $B$ is observed to depend on $h_{\max } / R$ only (Fig 6); that is,

$$
B=-1.687\left(\frac{h_{\max }}{R}\right)^{-0.62} .
$$

By combining Eqs. (6) and (7), we obtain

$$
\frac{H}{E^{*}}=0.5928\left(\frac{h_{\max }}{R}\right)^{0.62}\left(\frac{W_{u}}{W_{t}}\right) \text {. }
$$

Thus, the ratio of hardness to reduced modulus, $H / E^{*}$, can be obtained by determining $h_{\max } / R$ and $W_{u} / W_{t}$. Recently, an approximately linear relationship between $H / E^{*}$ and $W_{u} / W_{t}$ was obtained for conical and pyramidal indentation in elastic-plastic solids with work hardening. ${ }^{18,22,23}$ The two quantities were found to be proportional to each other with the proportionality factor a function of the indenter angle in conical indentation modeling and experiments. Equation (8) shows that, for the first time, a similar relationship exists for spherical indentation in elastic-plastic solids.

As an application of Eq. (8), we use it together with a well-known relationship between reduced modulus, $E^{*}$, initial unloading stiffness, $S$, and contact area, $A_{c},{ }^{5,24}$

$$
E^{*}=\frac{\sqrt{\pi}}{2} \frac{S}{\sqrt{A_{c}}} .
$$

By combining Eqs. (8) and (9) and the definition of hardness, $H=F_{\max } / A_{c}$, we obtained

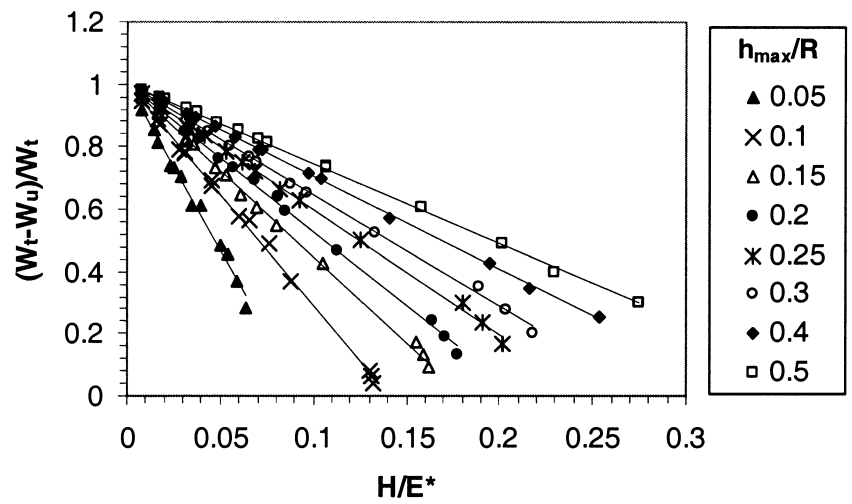

FIG. 5. For each fixed $h_{\max } / R$, a linear relationship exists between $H / E^{*}$ and $\left(W_{t}-W_{u}\right) / W_{t}$ for spherical indentation in elastic-plastic solids with work hardening. 


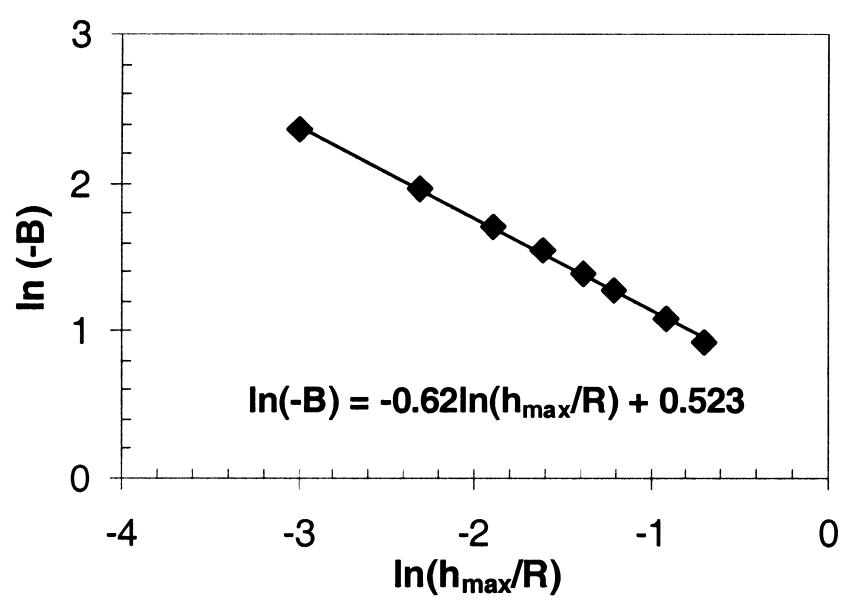

FIG. 6. A linear relationship between $\log \left(h_{\max } / R\right)$ and $\log (-B)$.

$$
\begin{aligned}
A_{c} & =\left[\frac{1.903 F_{\max }}{\left(\frac{h_{\max }}{R}\right)^{0.62}\left(\frac{W_{u}}{W_{t}}\right) S}\right]^{2}, \\
E^{*} & =\frac{0.4657 S^{2}\left(\frac{h_{\max }}{R}\right)^{0.62}\left(\frac{W_{u}}{W_{t}}\right)}{F_{\max }}, \\
H & =0.276\left(\frac{h_{\max }}{R}\right)^{1.24}\left(\frac{W_{u}}{W_{t}}\right)^{2} \frac{S^{2}}{F_{\max }} .
\end{aligned}
$$

Because $F_{\max }, h_{\max } / R, W_{u} / W_{t}$, and $S$ can be measured directly from the load-displacement curves, contact area, reduced modulus, and hardness can in principle be obtained from Eqs. (10)-(12). The main advantage of this energy-based method is that it applies to both piling-up and sinking-in of the surface profiles around indenters whereas the commonly used methods cannot be used when piling-up occurs. While this method is based on analysis of rigid spherical indentation in elastic-plastic solids with work hardening, it is necessary to test its robustness through experiments under realistic and often imperfect conditions, such as nonsphericity and nonrigidity of spherical indenters.

\section{EXPERIMENTS}

Spherical indentation experiments were conducted using a Nano Indenter XP (Oak Ridge, TN) from MTS with a rounded conical diamond indenter. The included cone angle was $90^{\circ}$ and the nominal tip radius was $10 \mu \mathrm{m}$. At least five indentations were made at each load to generate average values and standard deviations reported in this work. The load range was between 10 and $400 \mathrm{mN}$. The indentation experiments were conducted using load control with a constant loading rate. Unloading was initiated immediately after the load reached the prescribed maximum load at the end of each loading cycle without a holding period. All indentations were conducted at room temperature. The mechanical properties of tested materials, pure copper, 6061-T6 aluminum, pure tungsten, and fused silica, are shown in Table I. The composite reduced modulus, $E^{*}$, is given by

$$
\frac{1}{E^{*}}=\frac{1-v_{s}^{2}}{E_{s}}+\frac{1-v_{i}^{2}}{E_{i}}
$$

where $E_{s}$ and $v_{s}$ are the Young's modulus and Poisson's ratio of the samples, respectively. $E_{i}$ and $v_{i}$ are the Young's modulus and Poisson's ratio of the indenter, respectively. Specifically, $E_{i}=1141 \mathrm{GPa}$ and $\nu_{i}=0.07$ for the diamond indenter. The fused silica sample was obtained from MTS as the standard calibration material. The copper, aluminum, and tungsten samples were mechanically polished, finishing with 0.25 - $\mu \mathrm{m}$-diameter diamond paste. The average surface roughness, $R_{a}$, measured using a Wyco optical profilometer (Tucson, AZ), was 20, 49, and $23 \mathrm{~nm}$ for the polished copper, aluminum, and tungsten samples, respectively.

The relationship between $h_{f} / h_{\max }$ and $\left(W_{t}-W_{u}\right) / W_{t}$ for copper, aluminum, tungsten, and fused silica is shown in Fig. 4, together with finite element results. It is evident that, for all the materials tested, $h_{f} / h_{\max }$ is approximately proportional to $\left(W_{t}-W_{u}\right) / W_{t}$, which is consistent with the finite element analysis. The agreement suggests that this relationship is insensitive to the finite elasticity of the diamond indenter and imperfections in the diamond indenter geometry. However, the imperfection in the spherical shape of the diamond indenter can be shown to cause problems for direct applications of Eqs. (10)-(12). In the following, a method for obtaining an effective radius for imperfect spherical indenters is established to circumvent these problems.

For indenter shape calibration, instrumented spherical indentation experiments were conducted on the copper sample for depth ranging between $150 \mathrm{~nm}$ and $1850 \mathrm{~nm}$. The values for $S, F_{\max }, h_{\max }$, and $W_{u} / W_{t}$ were obtained

TABLE I. Mechanical properties of tested materials.

\begin{tabular}{lcccc}
\hline \hline & Young's & $\begin{array}{c}\text { Coisson's } \\
\text { modulus } \\
\text { Materials }\end{array}$ & $\begin{array}{c}\text { ratio } \\
\text { reduced } \\
\text { modulus }\end{array}$ & \\
\hline Copper & 126 & 0.345 & 127.1 & 25 \\
6061-T6 Aluminum & 70.4 & 0.347 & 74.8 & 26 \\
Tungsten & 409.8 & 0.28 & 320.3 & 26 \\
Fused silica & 72 & 0.17 & 69.6 & 27 \\
\hline \hline
\end{tabular}

The composite reduced modulus is calculated using Eq. (13) for the diamond indenter indenting the various materials. 
from load-displacement curves shown in Fig. 7. Equation (11) was then used to calculate the effective tip radius, $R_{e f f}$, at various depths, assuming a constant composite reduced modulus for copper, $E_{C u}^{*}=127.2 \mathrm{GPa}$. The relationship between $R_{\text {eff }}$ and $h_{\max }$ is shown in Fig. 8. Ideally, the radius should be independent of the indentation depth for a perfect spherical indenter. The effective tip radius was found, however, to be a function of indentation depth due to its imperfect geometry. An increase of more than $50 \%$ in tip radius over the depth was observed. A power-law fitting was used to interpolate the effective indenter radius. This indenter "shape function" is given by (Fig. 8)

$R_{\text {eff }}=1915.6 h_{\max }^{0.2755}$ for $150 \mathrm{~nm}<h_{\max }<1850 \mathrm{~nm}$.

Using the same diamond indenter, spherical indentation experiments were then conducted on 6061-T6 aluminum, tungsten, and fused silica with indentation depth from $180 \mathrm{~nm}$ to $1650 \mathrm{~nm}$. The load-displacement curves are shown in Fig. 9(a)-(c). The composite reduced modulus for aluminum, tungsten, and fused silica, calculated using Eq. (11) together with the shape function Eq. (14), at various depths is plotted in Fig. 10(a). As expected, the measured composite reduced modulus values are approximately depth independent. The measured composite reduced modulus of aluminum ranges from 74.3 to $78.6 \mathrm{GPa}$. The composite reduced modulus of tungsten ranges from 296 to $316.8 \mathrm{GPa}$. Compared with the calculated composite reduced modulus of aluminum and tungsten in Table I, the difference is within $8 \%$, suggesting that the proposed energy-based method together with indenter shape calibration is applicable to materials with a wide range of elastic modulus values. It also suggests that the proposed energy-based method can be used to analyze materials with Poisson's ratio ranging

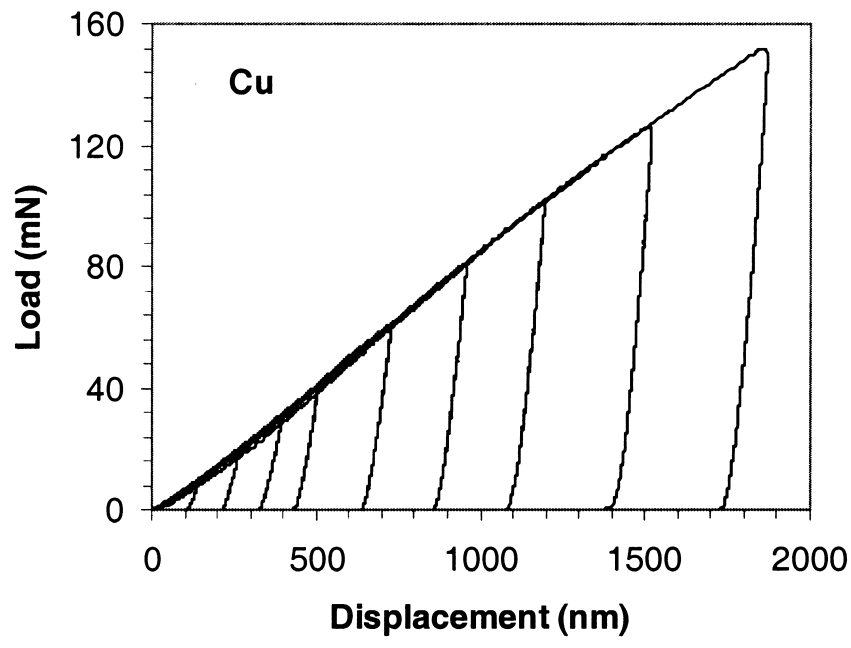

FIG. 7. Experimental load-displacement curves for spherical indentation in a copper sample.

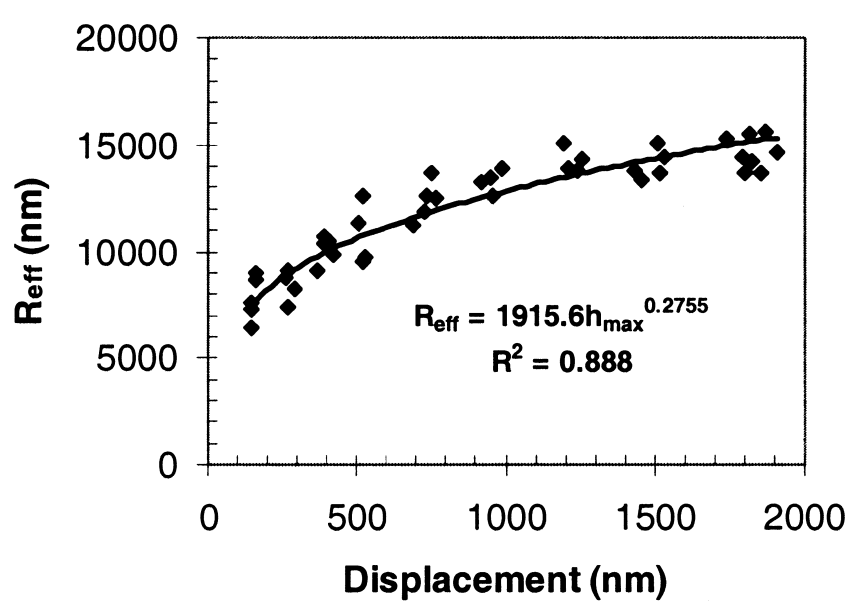

FIG. 8. Effective indenter radius, $R_{\text {eff }}$, as a function of indentation depth, $h_{\max }$, for an imperfect spherical diamond indenter.

from 0.2 to 0.35 , although Eqs. (10)-(12) were derived by using materials with $v=0.2$. We have performed additional finite element calculations to test the applicability of Eqs. (10), (11), and (12) to materials with $v=$ 0.3 . Specifically, we generated a set of loadingunloading curves for materials with $v=0.3$ and used Eq. (11), which was obtained for $v=0.2$, to evaluate the Young's modulus. The calculated Young's modulus value using Eq. (11) is within $15 \%$ of that used in the finite element input when the indentation depth is shallow with respect to the indenter radius (i.e., $h_{\max } / R<$ 0.2 ). We believe that in the derivation of Eqs. (10)-(12), most of the effect of Poisson's ratio has been taken into account by using the reduced modulus, $E^{*}=E /\left(1-v^{2}\right)$, although it has been shown that there is additional Poisson's ratio effect even in the well-known relationship Eq. (9) between reduced modulus, initial unloading stiffness, and contact area. ${ }^{28}$

The measured composite reduced modulus of fused silica is between 58.2 and $60.4 \mathrm{GPa}$. The difference between the calculated and measured value for fused silica is within $16 \%$. It should be noted that the fused silica data also exhibit the largest deviation in the correlation between $h_{f} / h_{\max }$ and $\left(W_{t}-W_{u}\right) / W_{t}$ (Fig. 4). Although the cause of this deviation is not yet known, it suggests that the relationship between $h_{f} / h_{\max }$ and $\left(W_{t}-W_{u}\right) / W_{t}$ may be used to help screen materials for which the new method is applicable. Specifically, the energy-based method consists of the following steps:

(i) Using Eqs. (11) and (13) to determine the effective indenter radius, $R_{e f f}$, as a function of indentation displacement, $h_{\max }$, by indenting a material with a known, depth-independent Young's modulus and Poisson's ratio. This shape function is an interpolation function over the indentation depth of interest and is not necessary to be the power-law form given by Eq. (14).

(ii) Checking the applicability of the energy-based 

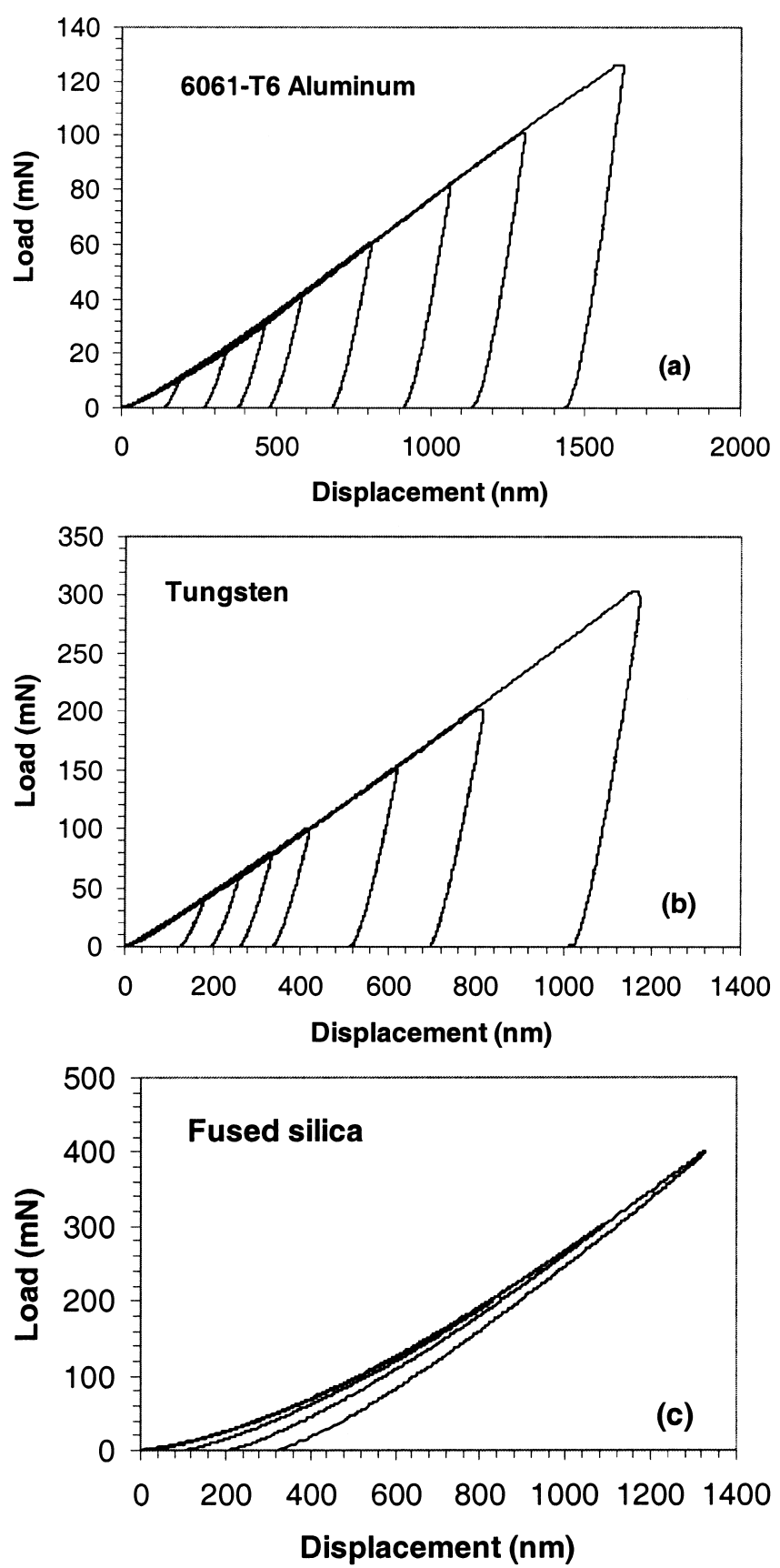

FIG. 9. Experimental load-displacement curves obtained from instrumented spherical indentation experiments in (a) aluminum, (b) tungsten, and (c) fused silica.

method by plotting $h_{f} / h_{\max }$ and $\left(W_{t}-W_{u}\right) / W_{t}$. A necessary condition is that the correlation exists for materials of interest.

(iii) Evaluating the reduced modulus for materials of interest using Eqs. (11) and (13) together with the shape function determined in step (i).

The robustness of this method is seen from the evaluation of the reduced modulus values for several materials using an imperfect spherical indenter with a varying effective radius of about $50 \%$. The method does not depend
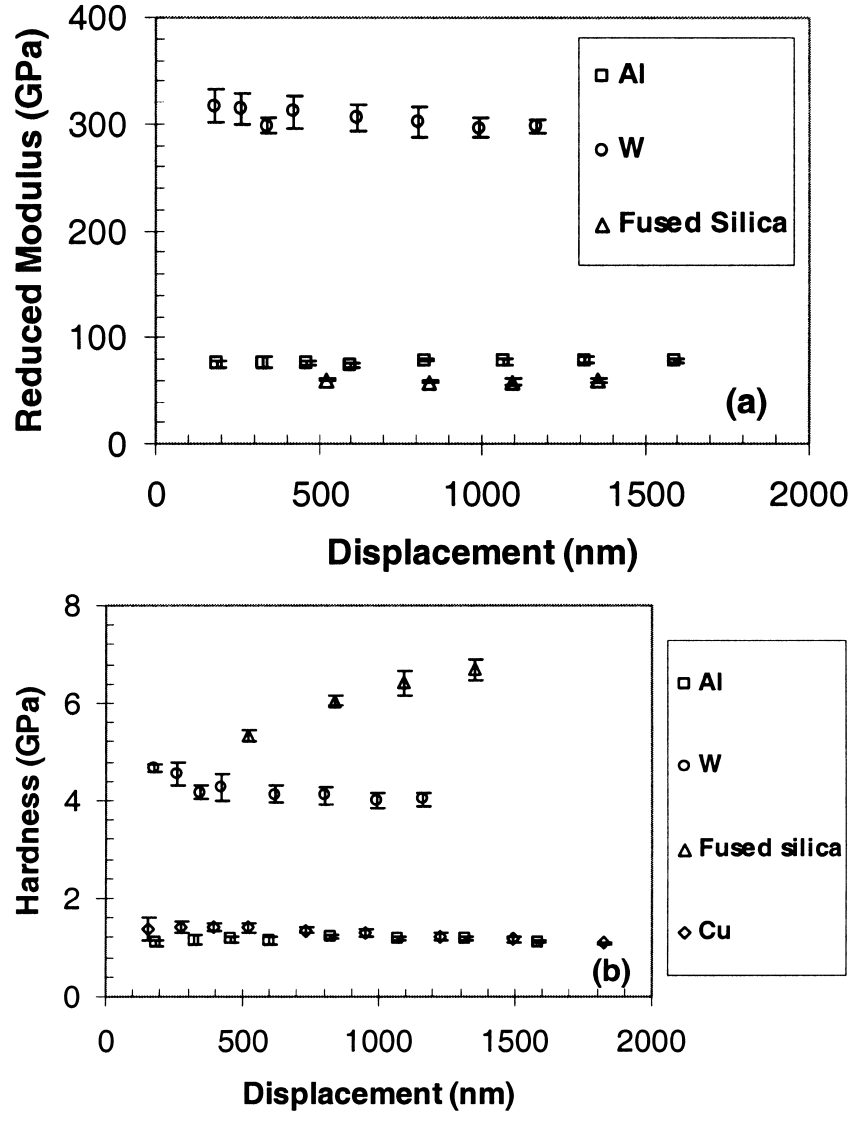

FIG. 10. (a) Measured composite reduced modulus and (b) hardness using the energy-based method together with indenter shape calibration. The error bar indicates the standard deviation of the measured values.

on assumptions about piling-up and sinking-in of materials around the spherical indenters.

Finally, hardness values for fused silica, W, Al, and $\mathrm{Cu}$ are obtained using Eq. (12) together with the indenter shape function Eq. (14). The results are shown in Fig. 10(b). While these values are within the range of reported hardness for the $\mathrm{Cu}, \mathrm{Al}$, and $\mathrm{W}$, detailed comparison is complicated because of several factors. Specifically, (i) most of the literature data were obtained using Berkovich indenters where a pronounced indentation size effect was reported for these materials. ${ }^{29-31}$ For sharp indentation, a strain-gradient plasticity model has been developed to describe the indentation size effect; that is, the increase of hardness with decreasing indentation depth. ${ }^{32}$ (ii) The indentation size effect is different under spherical indenters, where the hardness is not expected to show depth dependence but is expected to depend on the radius of spherical indenters according to analysis based on the theory of strain gradient plasticity. ${ }^{33}$ (iii) Hardness increases with indentation depth for spherical indentation in elastic-plastic solids with work hardening. ${ }^{2}$ With an imperfect spherical indenter, such as the one used in this work, it is possible that the two 
effects, work hardening and indentation size effect, cancel each other because of increasing $R_{\text {eff }}$ with depth, resulting in a slightly decreasing hardness for $\mathrm{Cu}, \mathrm{Al}$, and $\mathrm{W}$ with depth [Fig. 10 (b)]. While the imperfection in sphericity can be remedied for modulus measurements using a shape function [e.g., Eq. (14)], materials hardness is indenter shape dependent. It is therefore desirable to use as perfect spherical shape as possible for hardness measurements using spherical indenters.

\section{SUMMARY}

The degree of piling-up/sinking-in and the relationships between $H / E^{*},\left(W_{t}-W_{u}\right) / W_{t}$, and $h_{f} / h_{\max }$ have been studied using dimensional analysis and finite element calculations. For a fixed $h_{\max } / R$, a linear relationship exists between $H / E^{*}$ and $\left(W_{t}-W_{u}\right) / W_{t}$ and the proportionality depends on $h_{\max } / R$ only. Using the above relationships, together with the initial unloading stiffness, an energy-based method has been proposed to derive the contact area, reduced modulus, and hardness of materials from instrumented spherical indentation experiments. This new method can also be used to calibrate the effective tip radius of an imperfect spherical indenter. The validity of the new method was studied by instrumented indentation experiments on copper, aluminum, tungsten, and fused silica. Future work includes the refinement of the proposed energy method to cover a wider range of materials behavior.

\section{ACKNOWLEDGMENTS}

We would like to thank Yue Qi, T. Wes Capehart, Thomas A. Perry, Cameron Dash, and Mark W. Verbrugge for the help and valuable discussions.

\section{REFERENCES}

1. E10-01 Standard Test Method for BRINELL Hardness of Metallic Materials (ASTM International, West Conshohocken, 2003).

2. D. Tabor, The Hardness of Metals (Oxford University Press, London, U.K., 1951); Philosophical Magazine A 74, 1207 (1996).

3. B.W. Mott, Micro-indentation Hardness Testing (Butterworths, London, U.K., 1956).

4. A.C. Fischer-Cripps, Nanoindentation (Springer-Verlag, New York, 2002).

5. W.C. Oliver and G.M. Pharr, J. Mater. Res. 7, 1564 (1992).

6. J.S. Field and M.V. Swain, J. Mater. Res. 8, 297 (1993); J. Mater. Res. 10, 101 (1995).

7. F.M. Haggag and G.E. Lucas, Metall. Trans. 14A, 1607 (1983).

8. B. Taljat, T. Zacharia, and F.M. Haggaga, J. Mater. Res. 12, 965 (1997).

9. N. Huber and C. Tsakmakis, J. Mech. Phys. Solids 47, 1569, 1589 (1999).

10. J. Alcalá, A.E. Giannakopoulos, and S. Suresh, J. Mater. Res. 13, 1390 (1998).

11. S. Kucharski and Z. Mróz, J. Eng. Mater. Technol. 123, 235 (2001).
12. G. Herbert, G.M. Pharr, W.C. Oliver, B.N. Lucas, and J.L. Hay, Thin Solid Films 398-399, 331 (2001).

13. A.L. Norbury and T. Samuel, J. Iron Steel Inst. 117, 673 (1928).

14. M.M. Chaudhri and M. Winter, J. Phys. D.: Appl. Phys. 21, 370 (1988).

15. Y-T. Cheng and C-M. Cheng, Philos. Mag. Lett. 78, 115 (1998).

16. A. Bolshakov and G.M. Pharr, J. Mater. Res. 13, 1049 (1998).

17. Y-T. Cheng and C-M. Cheng, J. Appl. Phys. 84, 1284 (1998).

18. Y-T. Cheng, Z. Li, and C-M. Cheng, Philos. Mag. 82, 1821 (2002).

19. G.I. Barenblatt, Scaling, Self-similarity, and Intermediate Asymptotics (Cambridge University Press, Cambridge, 1996).

20. W. Ni, Y-T. Cheng, C-M. Cheng, and D.S. Grummon, GM Research Publication R\&D-9522 (April 23, 2003).

21. Y-T. Cheng and C-M. Cheng, J. Mater. Res. 14, 3493 (1999).

22. Y-T. Cheng and C-M. Cheng, Appl. Phys. Lett. 73, 614 (1998).

23. A.E. Giannakopoulos and S. Suresh, Scr. Mater. 40, 1191 (1999).

24. C-M. Cheng and Y-T. Cheng, Appl. Phys. Lett. 71, 2623 (1997).

25. K.A. Gschneidner, Solid State Physics 16, 275 (1964).

26. G. Simmons and H. Wang, Single Crystal Elastic Constants and Calculated Aggregate Properties: A Handbook, 2nd ed. (The M.I.T. Press, Cambridge, MA, 1971).

27. General Electric Fused Quartz Products Technical Data, general catalog number 7705-7725 (April 1985).

28. J.C. Hay, A. Bolshakov, and G.M. Pharr, J. Mater. Res. 14, 2296 (1999).

29. W.J. Poole, M.F. Ashby, and N.A. Fleck, Scr. Mater. 34, 559 (1996).

30. K.W. McElhaney, J.J. Vlassak, and W.D. Nix, J. Mater. Res. 13, 1300 (1998).

31. W.W. Gerberich, N.I. Tymiak, J.C. Grunlan, M.F. Horstemeryer, and M.I. Baskes, ASME J. Appl. Mech. 69, 433 (2002).

32. W.D. Nix and H. Gao, J. Mech. Phys. Solids 46, 411 (1998).

33. J.G. Swadener, E.P. George, and G.M. Pharr, J. Mech. Phys. Solids 50, 681 (2002).

\section{APPENDIX}

The total work during loading is given by integrating Eq. (2) from zero to $h_{\max }$. Although the integration cannot be made explicitly, the functional form can be obtained as follows,

$$
\begin{gathered}
W_{t}=\int_{0}^{h_{\max }} F_{l} d h=E R^{2} \int_{0}^{h_{\max }} \prod_{a}\left(Y / E, v, n, \frac{h}{R}\right) d h \\
\equiv E R^{3} \prod_{\tau}\left(\frac{Y}{E}, v, n, \frac{h_{\max }}{R}\right),
\end{gathered}
$$

where $\Pi_{\tau}$ is a dimensionless function such that

$$
R \frac{\partial}{\partial h} \prod_{\tau}\left(\frac{Y}{E}, \nu, n, \frac{h}{R}\right)=\prod_{\alpha}\left(Y / E, \nu, n, \frac{h}{R}\right) .
$$

During unloading, the force on the indenter $\left(F_{u}\right)$ is given by Eq. (4). In particular, the final indentation depth, $h_{f}$, is obtained by setting the left-hand side of Eq. (4) equal to zero and solving for $h_{f}$. Thus,

$$
h_{f}=R \prod_{\chi}\left(\frac{Y}{E}, v, n, \frac{h_{\max }}{R}\right),
$$


where $\Pi_{\chi}=h_{f} / R$, is a dimensionless function of $Y / E, \nu$, $n$, and $h_{\max } / R$. The reversible work during unloading is then given by

$$
\begin{gathered}
W_{u}=\int_{h_{f}}^{h_{\max }} F_{u} d h=E R^{2} \int_{h_{f}}^{h_{\max }} \prod_{\gamma}\left(\frac{Y}{E}, \nu, n, \frac{h}{R}, \frac{h_{\max }}{R}\right) d h \\
\equiv E R^{3} \prod_{\mu}\left(\frac{Y}{E}, \nu, n, \frac{h_{\max }}{R}\right),
\end{gathered}
$$

where $\Pi_{\mu}$ is a dimensionless function such that

$$
R \frac{\partial}{\partial h} \prod_{\mu}\left(\frac{Y}{E}, v, n, \frac{h}{R}\right)=\prod_{\gamma}\left(Y / E, \nu, n, \frac{h}{R}, \frac{h_{\max }}{R}\right) \text {. }
$$

The hardness, $H$, defined as the mean contact pressure under load, is given by

$$
H=\frac{F_{\max }}{A_{c}}=\frac{F_{\max }}{\pi a^{2}}=\frac{F_{\max }}{\pi\left(2 R h_{c}-h_{c}^{2}\right)},
$$

where $a$ is the contact radius. It is clear from Eqs. (2) and (3) that the ratio of $H$ to the reduced modulus, $E^{*} \equiv \mathrm{E} /$ $\left(1-v^{2}\right)$, is a function of $Y / E, v, n$, and $h_{\max } / R$; namely,

$$
\frac{H}{E^{*}}=\prod_{\varpi}\left(\frac{Y}{E}, v, n, \frac{h_{\max }}{R}\right) .
$$

Likewise, according to Eqs. (A1), (A2), and (A3), $\left(W_{t}-W_{u}\right) / W_{t}$ and $h_{f} / h_{\max }$ are also functions of $Y / E, \nu, n$, and $h_{\max } / R$. 